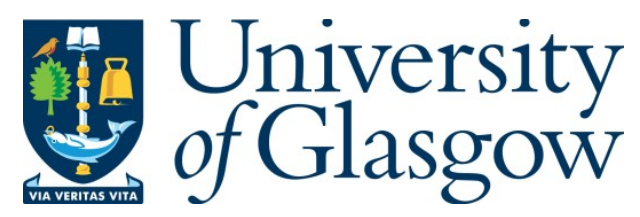

Rayel, O. K., Brante, G., Rebelatto, J. L., Souza, R. D., and Imran, M. A. (2014) Energy efficiency-spectral efficiency trade-off of transmit antenna selection. IEEE Transactions on Communications, 62(12), pp. 4293-4303.

There may be differences between this version and the published version. You are advised to consult the publisher's version if you wish to cite from it.

http://eprints.gla.ac.uk/132750/

Deposited on: 13 December 2016

Enlighten - Research publications by members of the University of Glasgow http://eprints.gla.ac.uk 


\title{
Energy Efficiency-Spectral Efficiency Trade-off of Transmit Antenna Selection
}

\author{
Ohara Kerusauskas Rayel, Student Member, IEEE, Glauber Brante, Member, IEEE \\ João Luiz Rebelatto, Member, IEEE, Richard Demo Souza, Senior Member, IEEE, \\ and Muhammad Ali Imran, Senior Member, IEEE
}

\begin{abstract}
We evaluate the Energy Efficiency-Spectral Efficiency (EE-SE) trade-off of Multiple-Input Multiple-Output (MIMO) and Transmit Antenna Selection/Maximum Ratio Combining (TAS/MRC) schemes. A realistic power consumption model (PCM) is considered, and it is shown that using TAS/MRC can provide significant energy savings when compared to MIMO in the low to medium spectral efficiency region, regardless the number of antenna elements. If the number of receive antennas is fixed, the energy efficiency gain of using TAS/MRC becomes even greater with the increasing number of transmit antennas. The optimal value of spectral efficiency that minimizes the energy consumption is obtained in closed-form, and confirmed by numerical results. Moreover, it is also shown that considering a non-realistic PCM can lead to mistakes when analyzing the gain obtained by using TAS/MRC instead of MIMO.
\end{abstract}

Index Terms-Transmit antenna selection, energy efficiency, spectral efficiency.

\section{INTRODUCTION}

The use of multiple transmit and receive antennas, or multiple-input multiple-output (MIMO) systems, has been shown to significantly improve the spectral efficiency (SE), which has been the main performance indicator for designing and optimizing wireless communication networks [1]. However, due to the increasing global concern about energy consumption, the energy efficiency (EE) became of particular interest [2]. From an EE point of view, the use of multiple antennas could lead to an increased energy consumption, since extra circuit and signal processing are required.

One of the most common assumptions in an energy efficient design is to quantify the network performance in terms of bits/Joule [3], [4], i.e., the maximum number of bits that can be delivered by the network normalized by the energy needed to deliver them. With the same goal, other metrics may also be assumed, such as the energy/bit to noise spectral density [5], or the throughput/energy of the network [6]. More importantly, a fundamental requirement for EE evaluation is to use an appropriate power consumption model (PCM). For instance, the authors in [7]-[9] have shown the impact of considering a realistic PCM in wireless sensor network scenarios, since, when the nodes are closer to each other, the consumption of the

This work has been partially supported by CNPq and CAPES (Brazil)

Ohara Kerusauskas Rayel, João Luiz Rebelatto, Glauber Brante and Richard Demo Souza are with CPGEI, UTFPR, Curitiba, PR, 80230-901, Brazil (email: \{oharakr, jlrebelatto, gbrante, richard\}@utfpr.edu.br).

Muhammad Ali Imran is with the Centre for Communication Systems Research (CCSR), University of Surrey, United Kingdom, GU2 7XH, (e-mail: m.imran@surrey.ac.uk)
RF circuit elements for transmitting and receiving may prevail over the power required for transmission. On the other hand, in the cellular networks context, the authors in [2], [10]-[12] have shown that a realistic PCM should also consider other important factors, such as the amplifier inefficiency, processing and backhauling power consumption, cooling, etc., since these factors have great impact in the overall system evaluation.

Moreover, it is known that SE and EE are usually conflicting objectives in a wireless system, so that a unified analysis must balance their relationship [13]. A pioneering analysis of the EE-SE trade-off in Rayleigh fading channels appears in [5], which received renewed interest in the last few years with the works of [14]-[19]. An important observation in [5] is that finding a closed-form expression for the $\mathrm{EE}$ as a function of the $\mathrm{SE}$ is quite challenging, since this task requires an inverse function of the capacity. Then, the authors proceed with an approximation for the low-SE regime of the EE-SE trade-off.

Recently, a novel closed-form approximation for the EESE trade-off was provided in [14], which turns out to be more general than that in [5]. Later, the case of independent and semi-correlated Rayleigh fading scenarios were considered in [15]. In that work, the authors also analyze the impact of considering a realistic PCM, while they derive closedform upper bounds for the EE-SE trade-off. Moreover, in [16], distributed MIMO and co-located MIMO are compared in terms of the EE-SE trade-off. The authors also consider different types of PCMs and show that distributed MIMO is more energy efficient than co-located MIMO for use at the cell edge. In addition, extensions to cooperative [17], [18] and automatic repeat request (ARQ) [19] scenarios were recently investigated from the EE-SE trade-off point of view.

In terms of energy efficiency, transmit antenna selection (TAS) appears as an interesting solution to reduce the circuitry consumption. In TAS, the receiver must inform the transmitter, via feedback, of which antenna has the best condition. Such technique achieves the same diversity order as other MIMO techniques employing all antennas at the transmitter [20], while decreases the energy consumption. As shown in [21], which compares TAS and transmit beamforming in terms of EE in a quasi-static fading scenario, TAS is a suboptimal strategy under the SE point of view, however, this fact is compensated by a higher EE. TAS is outperformed by beamforming only at considerably large distances (when the required transmit power prevails over the circuitry consumption). The use of TAS in large MIMO scenarios has been recently investigated in [22]. In that work, the goal is to 
improve the energy efficiency while considering two particular scenarios, when the circuit power is comparable to the transmit power, and when the circuit power is much smaller than the transmit power, so that it can be ignored. Results show that, if the circuit power is much larger than or comparable to the transmit power, using too many extra antennas can reduce $\mathrm{EE}$, so that a subset of antennas should be used for transmission. However, if the transmit power dominates the circuit power consumption, the EE increases monotonically with the number of selected antennas, so that all antennas should be employed. Nevertheless, the trade-off between SE and $\mathrm{EE}$ is not considered neither by [21], nor by [22].

In this paper we analyze the EE-SE trade-off of TAS/MRC, which, to the best of the author's knowledge, has not been analyzed in the literature yet. The challenge in this analysis is to find an expression for the inverse of the system capacity, which we solve by resorting to closed-form approximations. Moreover, we also obtain in closed-form the optimal spectral efficiency value that maximizes the energy efficiency of the TAS/MRC scheme, for a given number of antennas, which is supported by numerical results. Our analysis shows that, in the low to medium spectral efficiency region, TAS can be much more energy efficient than MIMO schemes that use all antennas, and that such advantage increases with the number of antennas. In addition, we consider a realistic double linear PCM, as in [14]. This model suits large-range communications systems, so that it includes a term that scales with the transmit power, and other that remains fixed. Our analysis shows that considerably different conclusions would be made by not considering a realistic PCM.

The remainder of this paper is organized as follows. In Section II we introduce the system model and the basics of MIMO systems capacity, the energy consumption model and the EE-SE trade-off formulation. In Section III we present the proposed EE-SE trade-off analysis of TAS/MRC, while some numerical results are discussed in Section IV. Finally, Section V concludes the paper.

Notations. We use bold upper case letters to denote matrices, like $\mathbf{H}$, and bold lower case letters to represent vectors, as $\mathbf{x}$, whose transpose conjugate is denoted by $\mathbf{x}^{\dagger} \cdot \log (\cdot)$ is the natural $\log$ arithm, $\log _{2}(\cdot)$ is the base- 2 logarithm, and $\mathbb{E}[\cdot]$ is the mathematical expectation. The probability density function (pdf) of a random variable $z$ is represented by $p_{z}(z)$.

\section{PRELIMINARIES}

\section{A. System Model}

This work considers a wireless communication system where each transmitter is assumed to be equipped with $t$ transmit antennas, while the receiver has $r$ receive antennas. Omitting the time index, the received signal is given by

$$
\mathbf{y}=\sqrt{\kappa} \mathbf{H} \mathbf{x}+\mathbf{n},
$$

where $\mathbf{x} \in \mathbb{C}^{t \times 1}$ and $\mathbf{y} \in \mathbb{C}^{r \times 1}$ are the $t$ transmitted and $r$ received vectors, respectively, and $\mathbf{n} \in \mathbb{C}^{r \times 1}$ is zero-mean additive white Gaussian noise (AWGN) with variance $N_{0}$ per dimension. The total power of the transmitted signal vector is denoted by $P_{\mathrm{Tx}}=\mathbb{E}\left[\mathbf{x}^{\dagger} \mathbf{x}\right]$, while the path-loss between the transmitter and the receiver is denoted by $\kappa$. The matrix $\mathbf{H} \in \mathbb{C}^{r \times t}$ contains the channel fading coefficients $h_{i, j}$ from transmit antenna $j$ to receive antenna $i$, which are complex Gaussian with zero mean and unity variance and assumed to be independent and identically distributed (i.i.d.) across space and time. Moreover, it is considered that only the receivers have perfect channel state information (CSI). Finally, let us also define $n=\max \{t, r\}, m=\min \{t, r\}$.

Then, the average signal-to-noise ratio (SNR) per receive antenna can be expressed as

$$
\bar{\gamma}=\frac{\kappa P_{\mathrm{Tx}}}{N_{0} B},
$$

with $B$ representing the channel bandwidth (in Hertz).

1) System Capacity for SISO: Assume for the moment a single-input single-output (SISO) system, where both transmitter and receiver are single antenna devices $(t=r=1)$. The instantaneous SNR, subject to channel realization, is

$$
\gamma=|h|^{2} \bar{\gamma}
$$

where $h=h_{1,1}$. Moreover, the pdf of $\gamma$ is [23]

$$
p_{\gamma}(\gamma)=\frac{e^{-\gamma / \bar{\gamma}}}{\bar{\gamma}}
$$

Therefore, the channel capacity is given by [24]

$$
\begin{aligned}
C_{\text {SISO }} & =\int_{0}^{\infty} B \log _{2}\left(1+|h|^{2} \bar{\gamma}\right) p_{\gamma}(\gamma) d \gamma \\
& =\int_{0}^{\infty} B \log _{2}(1+\gamma) \frac{e^{-\gamma / \bar{\gamma}}}{\bar{\gamma}} d \gamma .
\end{aligned}
$$

By using the following integral solution [25, §4.222.8]

$$
\begin{aligned}
& \int_{0}^{\infty} \log (1+a x) x^{b} e^{-x} d x= \\
& \sum_{j=0}^{b} \frac{b !}{(b-j) !}\left[\frac{(-1)^{b-j-1}}{a^{b-j}} e^{\frac{1}{a}} \mathrm{E}_{i}\left(-\frac{1}{a}\right)+\right. \\
& \left.\sum_{k=1}^{b-j} \frac{(k-1) !}{(-a)^{b-j-k}}\right],
\end{aligned}
$$

where $\mathrm{E}_{i}(x)=\int_{-\infty}^{x} \frac{e^{t}}{t} d t$ is the exponential integral function (Cauchy principal value integral definition), and by doing $x=$ $\gamma / \bar{\gamma}, d x=d \gamma / \bar{\gamma}, a=\bar{\gamma}$ and $b=0$, we obtain the SISO system capacity as

$$
C_{\mathrm{SISO}}=-B \frac{e^{1 / \bar{\gamma}}}{\log (2)} \mathrm{E}_{i}\left(-\frac{1}{\bar{\gamma}}\right) .
$$

2) System Capacity for MIMO: For a MIMO system as specified by (1), the capacity can be obtained by doing

$$
C_{\text {MIMO }}=\mathbb{E}\left[B \log _{2} \operatorname{det}\left(\mathbf{I}_{m}+\frac{\bar{\gamma}}{t} \boldsymbol{\Xi}\right)\right],
$$

where $\mathbf{I}_{m}$ is an $m \times m$ identity matrix, and $\boldsymbol{\Xi} \in \mathbb{C}^{m \times m}$ is a random matrix given by

$$
\boldsymbol{\Xi}=\left\{\begin{array}{ll}
\mathbf{H H}^{\dagger} & t \geq r \\
\mathbf{H}^{\dagger} \mathbf{H} & t<r
\end{array} .\right.
$$


In the case of i.i.d. Rayleigh fading channels, the MIMO system capacity yields [15]

$$
C_{\mathrm{MIMO}}=B \log _{2}\left[m !\left(\frac{\bar{\gamma}}{t}\right)^{m} \mathcal{L}_{m}^{n-m}\left(-\frac{t}{\bar{\gamma}}\right)\right],
$$

where $\mathcal{L}_{m}^{\alpha}(x)$ is the Laguerre polynomial of order $m$ [25, $\S 8.970 .1]$

$$
\mathcal{L}_{m}^{\alpha}(x)=\frac{1}{m !} e^{x} x^{-\alpha} \frac{d^{m}}{d x^{m}}\left(e^{-x} x^{m+\alpha}\right) .
$$

Finally, for the high SNR region, it was shown in [15] that the capacity in (10) can be approximated as

$$
C_{\mathrm{MIMO}} \approx B \log _{2}\left[\left(\frac{\bar{\gamma}}{t}\right)^{m} \frac{n !}{(n-m) !}\right] .
$$

\section{B. Energy Consumption Model}

In practice, the total power consumed by a transmitter (referred as $P_{\text {Tot }}$ ) is composed not only of the transmission power, but also of the power consumption related to the circuitry hardware. Thus, in this work, we adopt the more realistic double linear power consumption model in order to evaluate the EE-SE trade-off. According to the aforementioned model, the total power consumption is [14]

$$
P_{\mathrm{Tot}}=t\left(\Delta_{P} P_{t}+P_{0}\right)+P_{1},
$$

where $P_{t}=P_{\mathrm{Tx}} / t$ is the per antenna transmit power, $P_{0}$ is the part of the power that grows linearly with the number of transmit antennas, $P_{1}$ is the part that does not depend on the number of transmit antennas and thus remains fixed. Alternatively, we can re-write (13) as a sum of two terms, $P_{\text {Tot }}=P_{\mathrm{V}}+P_{\mathrm{F}}$, where $P_{\mathrm{V}}=t \Delta_{P} P_{t}$ is the part that varies with the transmit power, while $P_{\mathrm{F}}=t P_{0}+P_{1}$ remains fixed with respect to $P_{t}$.

\section{Energy Efficiency-Spectral Efficiency (EE-SE) Trade-off}

As introduced in [5], the concept of EE-SE trade-off expresses the EE as a function of the SE. Let $C$ (bits/s) be the maximum rate of information that can be reliably transmitted by a given system and $P_{\text {Tot }}$ be the total consumed power for transmitting data at this rate, then the EE can be defined as

$$
E=\frac{C}{P_{\text {Tot }}},
$$

which is the bit-per-Joule capacity of the system. Through the Shannon's capacity theorem [24], the maximum achievable SE, or equivalently the ergodic channel capacity per unit bandwidth (in bits/s/Hz), can be expressed as

$$
S=\frac{C}{B}=f(\bar{\gamma})
$$

so that $f: \bar{\gamma} \in[0,+\infty) \rightarrow S \in[0,+\infty)$.

Next, we express the EE as a function of SE for the baseline SISO and MIMO systems.
1) EE Upper Bound for SISO: Let us upper bound the energy efficiency of a SISO system by considering that Shannon's capacity can be achieved. Moreover, from now on we refer to the upper bound of EE for a SISO system as $E_{\mathrm{SISO}}$. Then, we can re-write the SISO bit-per-Joule capacity as

$$
E_{\mathrm{SISO}}=\frac{S_{\mathrm{SISO}} B}{P_{\mathrm{Tot}}}=\frac{C_{\mathrm{SISO}}}{\Delta_{P} P_{\mathrm{SISO}}+P_{0}+P_{1}},
$$

recalling that $S_{\mathrm{SISO}}$ is the achievable SE of SISO, $P_{\mathrm{SISO}}$ is the transmit power used for the SISO scheme, and that there is only $t=1$ transmit antenna.

Moreover, we can see from (2) that $P_{\text {SISO }}=\frac{N_{0} B}{\kappa} \bar{\gamma}$, while $\bar{\gamma}=f^{-1}\left(C_{\text {SISO }}\right)$, which leads to

$$
E_{\mathrm{SISO}}=\frac{C_{\mathrm{SISO}}}{\Delta_{P} \frac{N_{0} B}{\kappa} f^{-1}\left(C_{\mathrm{SISO}}\right)+P_{0}+P_{1}} .
$$

However, let us remark that a closed-form expression for $E_{\text {SISO }}$ is hard to obtain, since $f^{-1}\left(C_{\text {SISO }}\right)$ requires the inverse of the exponential integral in (7).

2) EE Upper Bound for MIMO: Similarly, we can upper bound the energy efficiency of the MIMO system by doing

$$
\begin{aligned}
E_{\mathrm{MIMO}} & =\frac{S_{\mathrm{MIMO}} B}{t\left(\Delta_{P} P_{t}+P_{0}\right)+P_{1}} \\
& =\frac{C_{\mathrm{MIMO}}}{t\left(\Delta_{P} \frac{N_{0} B}{\kappa} f^{-1}\left(C_{\mathrm{MIMO}}\right)+P_{0}\right)+P_{1}} .
\end{aligned}
$$

However, a closed-form expression for $E_{\mathrm{MIMO}}$ is also hard to obtain, since (10) involves the Laguerre polynomial of order $m$. Nevertheless, we resort to the high SNR approximation in (12), so that

$$
f^{-1}\left(C_{\mathrm{MIMO}}\right) \approx t\left[2^{S_{\mathrm{MIMO}}} \frac{(n-m) !}{n !}\right]^{\frac{1}{m}} .
$$

\section{EE-SE TRADE-OFF FOR TAS/MRC}

\section{A. TAS/MRC Channel Capacity}

When the Transmit Antenna Selection/Maximum Ratio Combining (TAS/MRC) scheme is employed, only 1 out of $t$ transmit antennas is selected per transmission block, so that $P_{t}=P_{\mathrm{Tx}}$ for this particular antenna. Thus, the system effective SNR can be written as [23]

$$
\gamma_{\Sigma}=\bar{\gamma} \max _{j} \sum_{i=1}^{r}\left|h_{i, j}\right|^{2}
$$

where the maximum over $j$ represents that the best out of the $t$ transmit antennas is selected, while the sum is the MRC output of the $r$ receive antennas.

Then, the average TAS/MRC channel capacity is given by $C_{\mathrm{TAS} / \mathrm{MRC}}=\mathbb{E}\left[B \log _{2}\left(1+\gamma_{\Sigma}\right)\right]$. However, since the solution of this equation is not trivial, we resort to the same tight approximation used in [26] for the logarithm expectation, so that the TAS/MRC channel capacity can be approximated as

$$
C_{\mathrm{TAS} / \mathrm{MRC}} \approx \tilde{B}\left[\log \left(1+\mu_{\gamma}\right)-\frac{\sigma_{\gamma}^{2}}{2\left(1+\mu_{\gamma}\right)^{2}}\right],
$$

where $\tilde{B}=B \log _{2} e, \mu_{\gamma}$ and $\sigma_{\gamma}$ are the first moment and the standard deviation of $\gamma_{\Sigma}$, respectively. 
The pdf of the instantaneous SNR seen at the receiver in a system operating under TAS/MRC, with a given average SNR $\bar{\gamma}$, is given by [26]

$$
p_{\gamma_{\Sigma}}(\gamma)=\frac{t e^{-\frac{\gamma}{\bar{\gamma}}}}{(r-1) \bar{\gamma}}\left(\frac{\gamma}{\bar{\gamma}}\right)^{r-1}\left[1-e^{-\frac{\gamma}{\gamma}} \sum_{n=0}^{r-1} \frac{1}{n !}\left(\frac{\gamma}{\bar{\gamma}}\right)^{n}\right]^{t-1}
$$

Therefore, the first moment of $\gamma_{\Sigma}$ is

$$
\mu_{\gamma}=\int_{0}^{\infty} \gamma_{\Sigma} p_{\gamma_{\Sigma}}(\gamma) d \gamma_{\Sigma}=K_{1} \cdot \bar{\gamma}
$$

where $K_{1}$ is a constant given by

$$
\begin{aligned}
K_{1} & =\frac{t}{(r-1) !} \sum_{m=0}^{t-1}\left[(-1)^{m}\left(\begin{array}{c}
t-1 \\
m
\end{array}\right)\right. \\
& \left.\times \sum_{n=0}^{m(r-1)} a_{n}(r, m) \frac{(r+n) !}{(m+1)^{r+n+1}}\right],
\end{aligned}
$$

with $a_{n}(r, m)$ being the coefficient of $x^{n}, n \in[0, m(r-1)]$, in the expansion of $\left(\sum_{k=0}^{r-1} x^{k} / k !\right)^{m}$.

Similarly, the second moment of $\gamma_{\Sigma}$ is

$$
E\left(\gamma^{2}\right)=\int_{0}^{\infty} \gamma_{\Sigma}^{2} p_{\gamma_{\Sigma}}(\gamma) d \gamma_{\Sigma}=K_{2} \bar{\gamma}^{2}
$$

where the constant $K_{2}$ is

$$
\begin{aligned}
K_{2} & =\frac{t}{(r-1) !} \sum_{m=0}^{t-1}\left[(-1)^{m}\left(\begin{array}{c}
t-1 \\
m
\end{array}\right)\right. \\
& \left.\times \sum_{n=0}^{m(r-1)} a_{n}(r, m) \frac{(r+n+1) !}{(m+1)^{r+n+2}}\right] .
\end{aligned}
$$

Then, the variance of $\gamma_{\Sigma}$ can be simply obtained by combining (25) and (23) as

$$
\sigma_{\gamma}^{2}=E\left(\gamma^{2}\right)-\mu_{\gamma}^{2}
$$

Nevertheless, even though plugging (27) into (21) expresses a good approximation to the capacity of a TAS/MRC scheme, it remains difficult (if possible) to find its inverse with respect to $\bar{\gamma}$. Thus, aiming at a closed-form expression for the inverse function of $f^{-1}\left(C_{\mathrm{TAS} / \mathrm{MRC}}\right)$, we define the following lemma.

Lemma 1 . The TAS/MRC capacity can be expressed as

$$
\begin{aligned}
C_{\mathrm{TAS} / \mathrm{MRC}} & \approx \tilde{B}\left[\log \left(\mu_{\gamma}\right)-\frac{\sigma_{\gamma}^{2}}{2 \mu_{\gamma}^{2}}\right] \\
& =\tilde{B}\left[\log \left(K_{1} \bar{\gamma}\right)-\frac{K_{2}-K_{1}^{2}}{2 K_{1}^{2}}\right] .
\end{aligned}
$$

Proof: Exploiting the fact that $1+\mu_{\gamma} \approx \mu_{\gamma}$ for larger values of $\mu_{\gamma}$, we further approximate (21), which yields (28).

\section{B. EE Upper Bound for TAS/MRC}

Theorem 1. The upper bound for the EE of the TAS/MRC scheme is given by

$$
E_{\mathrm{TAS} / \mathrm{MRC}}=\frac{C_{\mathrm{TAS} / \mathrm{MRC}}}{\Delta_{P} \frac{N_{0} B}{\kappa K_{1}} \exp \left(\frac{C_{\mathrm{TAS} / \mathrm{MRC}}}{\bar{B}}+\frac{K_{2}-K_{1}^{2}}{2 K_{1}^{2}}\right)+P_{0}+P_{1}} .
$$

Proof: From (28), we can isolate the average SNR $\bar{\gamma}$ as $\bar{\gamma}=f^{-1}\left(C_{\mathrm{TAS} / \mathrm{MRC}}\right) \approx \frac{1}{K_{1}} \exp \left(\frac{C_{\mathrm{TAS} / \mathrm{MRC}}}{\tilde{B}}+\frac{K_{2}-K_{1}^{2}}{2 K_{1}^{2}}\right)$.

Then, we can upper bound the bit-per-Joule capacity of the TAS/MRC scheme as

$$
E_{\mathrm{TAS} / \mathrm{MRC}}=\frac{C_{\mathrm{TAS} / \mathrm{MRC}}}{t\left(\Delta_{P} \frac{N_{0} B}{\kappa} f^{-1}\left(C_{\mathrm{TAS} / \mathrm{MRC}}\right)+P_{0}\right)+P_{1}},
$$

which, by plugging (30) and using the fact that $t=1$ for TAS, since only one transmit antenna is selected, leads to (29) completing the proof.

\section{Energy Efficiency Optimization}

Theorem 2. The optimal spectral efficiency, which maximizes the energy efficiency for a given number of antennas, is

$S_{\text {TAS/MRC }}^{\star}=\log _{2} e \cdot\left[W\left(e^{-\frac{K_{2}-K_{1}^{2}}{2 K_{1}^{2}}-1} \frac{\left(P_{0}+P_{1}\right) \kappa K_{1}}{\Delta_{P} N_{0} B}\right)+1\right]$,

where $W(\cdot)$ corresponds to the Lambert-W function [27].

Proof: Taking the first order derivative of (29) with respect to $C_{\text {TAS/MRC yields }}$

$$
\begin{aligned}
& \frac{d E_{\mathrm{TAS} / \mathrm{MRC}}}{d C_{\mathrm{TAS} / \mathrm{MRC}}}= \\
& \frac{\Delta_{P} \frac{N_{0} B}{\kappa K_{1}}\left(\tilde{B}-C_{\mathrm{TAS} / \mathrm{MRC}}\right) e^{\frac{C_{\mathrm{TAS} / \mathrm{MRC}}}{\bar{B}}+\frac{K_{2}-K_{1}^{2}}{2 K_{1}^{2}}}+\tilde{B}\left(P_{0}+P_{1}\right)}{\tilde{B}\left(\Delta_{P} \frac{N_{0} B}{\kappa K_{1}} e^{\frac{C_{\mathrm{TAS} / \mathrm{MRC}}}{\bar{B}}+\frac{K_{2}-K_{1}^{2}}{2 K_{1}^{2}}}+P_{0}+P_{1}\right)^{2}} .
\end{aligned}
$$

Then, by setting $\frac{d E_{\mathrm{TAS} / \mathrm{MRC}}}{d C_{\mathrm{TAS} / \mathrm{MRC}}}=0$ and isolating $C_{\mathrm{TAS} / \mathrm{MRC}} / B$, we obtain the closed form expression for the optimal SE that maximizes the EE, as presented in (32).

\section{Energy Efficiency Gain of TAS/MRC over MIMO System}

In order to evaluate when the TAS/MRC scheme outperforms the MIMO scheme in terms of energy efficiency, following [14] we define the energy efficiency gain as

$$
G_{\mathrm{EE}}=\frac{E_{\mathrm{TAS} / \mathrm{MRC}}}{E_{\mathrm{MIMO}}} .
$$

If an ideal theoretical PCM is considered, where only the transmit power is taken into account, this gain is calculated as

$$
G_{\mathrm{EE}, \mathrm{Th}}=\frac{P_{\mathrm{MIMO}}}{P_{\mathrm{TAS} / \mathrm{MRC}}} \text {. }
$$

\section{E. Circuit Consumption Optimization}

In the double linear PCM, $P_{0}$ is the part of the circuit consumption power that grows linearly with the number of antennas and $P_{1}$ remains fixed. Obviously if we minimize the $P_{0} / P_{1}$ ratio, we lower the overall power consumption and consequently maximize the energy efficiency. However, let us remark that this ratio is highly dependent on the hardware design, size of the nodes, cooling requirements and power amplifier design and can only be varied by redesigning these 


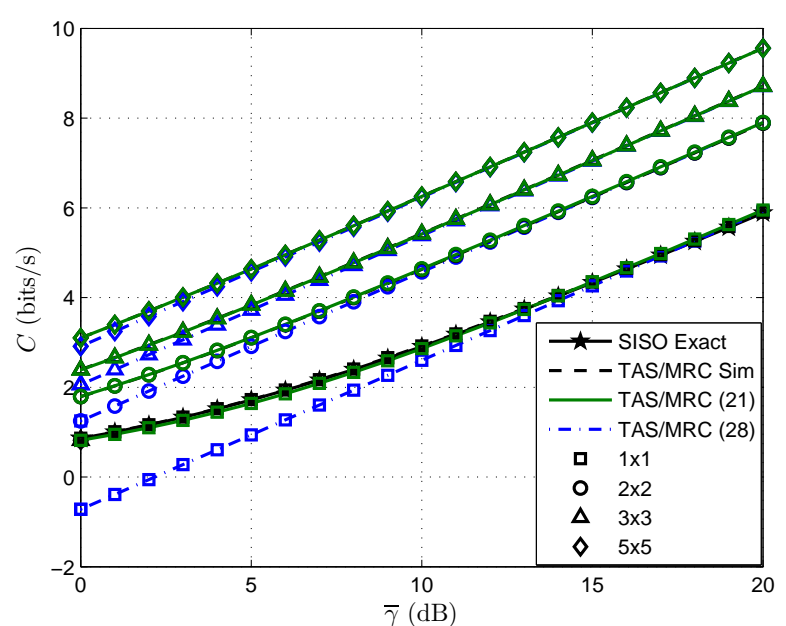

Fig. 1. Monte Carlo simulated (Sim) exact capacity and the approximated capacity, as given by (28) and (21), of TAS/MRC as a function of the average SNR between TX and RX.

aspects. Nevertheless, it is worth mentioning that, although changing this ratio is not very straightforward in practical system, this analysis can be helpful as a design guideline for setting desirable targets for the hardware designers.

\section{NUMERICAL RESULTS}

In this section, we present some numerical results in order to evaluate the previous analysis. In conformity to [14], the considered systems parameters are shown in Table I.

TABLE I

SYSTEM PARAMETERS

\begin{tabular}{ccc|ccc}
\hline$N_{0}$ & 1 & $\mathrm{~W} / \mathrm{Hz}$ & $P_{0}$ & $0-600$ & $\mathrm{~W}$ \\
$B$ & 1 & $\mathrm{~Hz}$ & $P_{1}$ & 225 & $\mathrm{~W}$ \\
$\kappa$ & 1 & & $\Delta_{P}$ & 7.25 & \\
\hline
\end{tabular}

From Fig. 1 it can be seen that the proposed approximation in (28) for the TAS/MRC capacity gets closer to (21) when the number of antennas increases (different antenna setups are considered: $1 \times 1,2 \times 2,3 \times 3$ and $5 \times 5)$. We also show that the approximation in (21) agrees very well with the exact capacity obtained by Monte Carlo simulations. In Fig. 2 the accuracy of the TAS/MRC capacity approximation made in (28) is evaluated in terms of the energy efficiency upper bound, by comparing it to the EE upper bound obtained by numerically inverting the capacity expression in (21). It can be seen that, as the number of antennas increases, the proposed approximation improves.

Fig. 3 presents the energy efficiency versus the spectral efficiency for SISO, MIMO and TAS/MRC schemes. It can be seen that MIMO becomes more energy efficient than TAS/MRC for high spectral efficiency scenarios, while TAS/MRC has advantage in the low spectral efficiency region, since only one transmit antenna is selected at a time, and just one transmit circuit and power amplifier is turned on per transmission. The ratio between the fixed to total power ratio $P_{\mathrm{F}} / P_{\text {Tot }}$ for the TAS/MRC system at the threshold where

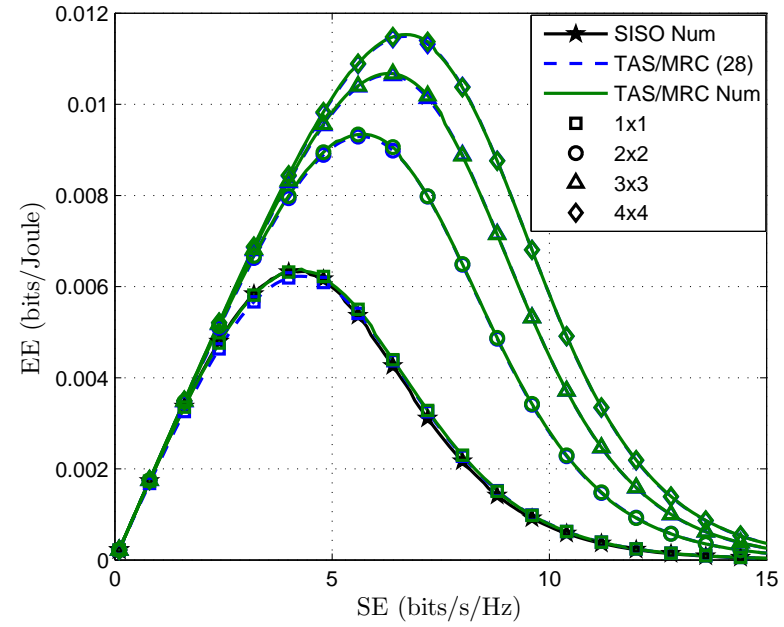

Fig. 2. Energy efficiency upper bound for the TAS/MRC scheme, varying the number of antennas, based on numerically inverting (Num) the capacity expression in (21) and on the proposed formulation in (28), and for a SISO configuration obtained by numerically inverting (7).

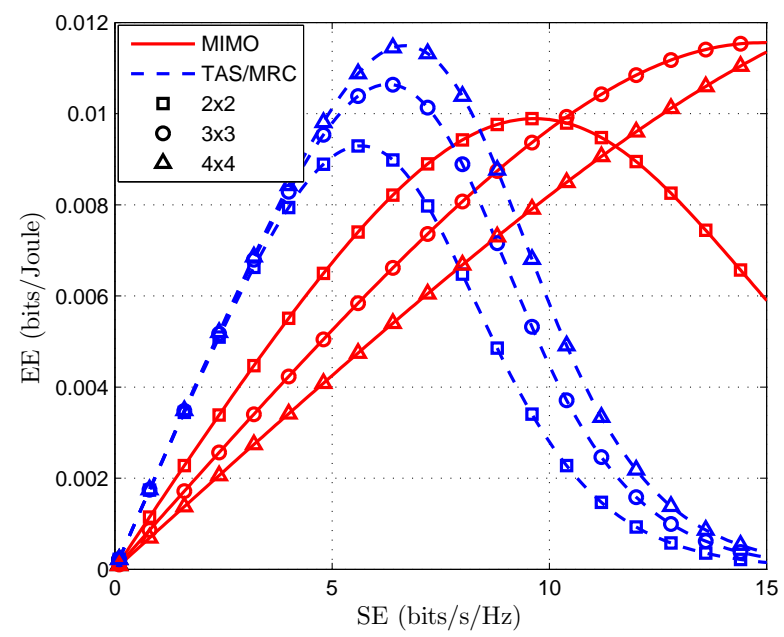

Fig. 3. Energy efficiency upper bound for TAS/MRC and MIMO schemes, based on the approximate transmit power, varying the number of antennas.

MIMO becomes more energy efficient are $57.27 \%, 45.37 \%$ and $36.95 \%$ for the $2 \times 2,3 \times 3$ and $4 \times 4$ configurations, respectively. We can see that, as the number of antennas increases, this ratio decreases, since the power amplifier consumption becomes dominant at the high spectral efficiency region.

The same analysis of Fig. 3 is repeated in Fig. 4, but with a fixed small number of $r=2$ receive antennas, what is a more practical assumption regarding the user equipment. Moreover, we define $M=t \cdot r$ and we try to preserve similar values of $M$ for both figures, i.e., the configurations $2 \times 2(M=4), 3 \times 3$ $(M=9)$ and $4 \times 4(M=16)$ of Fig. 3 will be compared to the configurations $2 \times 2(M=4), 4 \times 2(M=8)$ and $8 \times 2(M=16)$ in Fig. 4 . The results show that the difference between TAS/MRC and MIMO in terms of energy efficiency becomes even greater when $r$ is fixed. The ratio between the fixed to total power ratio $P_{\mathrm{F}} / P_{\text {Tot }}$ for the TAS/MRC system at the threshold where MIMO becomes more energy efficient is $43.41 \%$ with the $4 \times 2$ configuration, and $19.04 \%$ for the $8 \times 2$ configuration. Comparing Fig. 3 with Fig. 4, we observe 


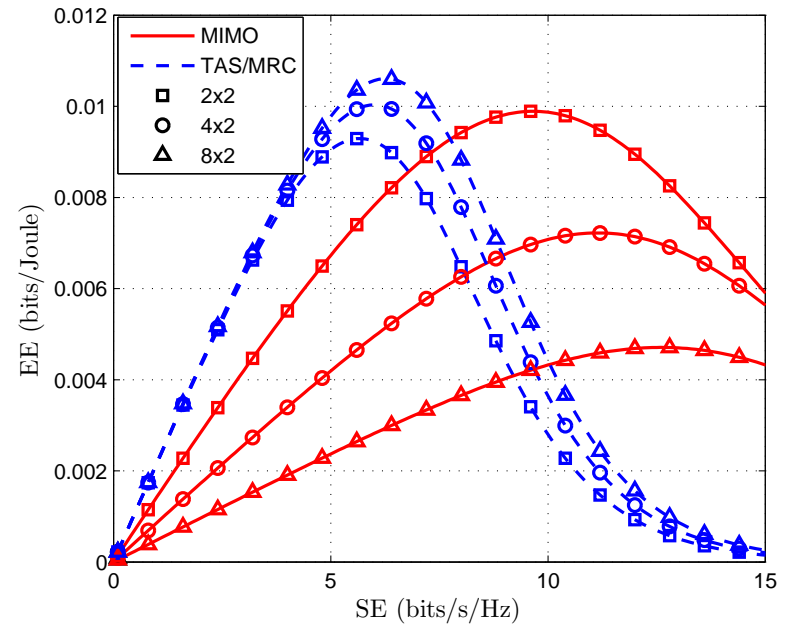

Fig. 4. Energy efficiency upper bound for TAS/MRC and MIMO schemes, based on the approximate transmit power, varying the number of antennas, with $r$ fixed.

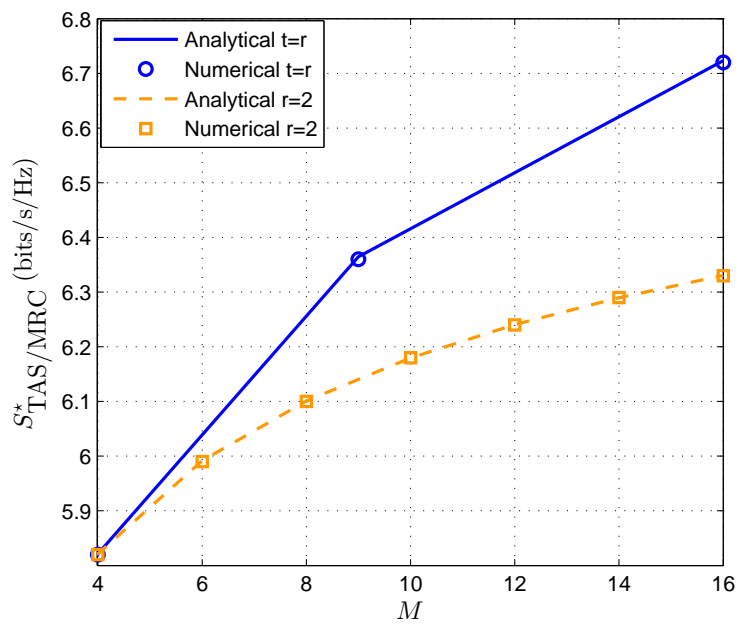

Fig. 5. Optimal spectral efficiency value that maximizes the energy efficiency of the TAS/MRC System, for various number of antenna elements.

that MIMO is less energy efficient when $r=2$ than when $t=r$ for a fixed value of $M$, and that the the threshold where TAS/MRC becomes less energy efficient than MIMO occurs in a higher spectral efficiency value, which demands higher transmit power (increasing $P_{\mathrm{V}}$ ), decreasing the $P_{\mathrm{F}} / P_{\text {Tot }}$ ratio.

Fig. 5 shows that the optimal EE analitically obtained in (32) precisely matches the numerical results. Such a result can be used as a designing tool for a network operating under the TAS/MRC scheme, since the optimal number of antennas that maximizes the energy efficiency can be selected previously for a given spectral efficiency. Alternatively, for a given number of antennas, it is also possible to obtain from (32) the spectral efficiency value that optimizes the energy efficiency.

The gain in energy efficiency of the TAS/MRC scheme over the MIMO scheme for various configurations of antennas is presented in Fig. 6, considering the theoretical PCM, where only the transmit power is taken into account. As expected, the gain decreases as the spectral efficiency increases. A similar analysis is presented in Fig. 7, however, considering the double linear PCM, where the power consumed by the

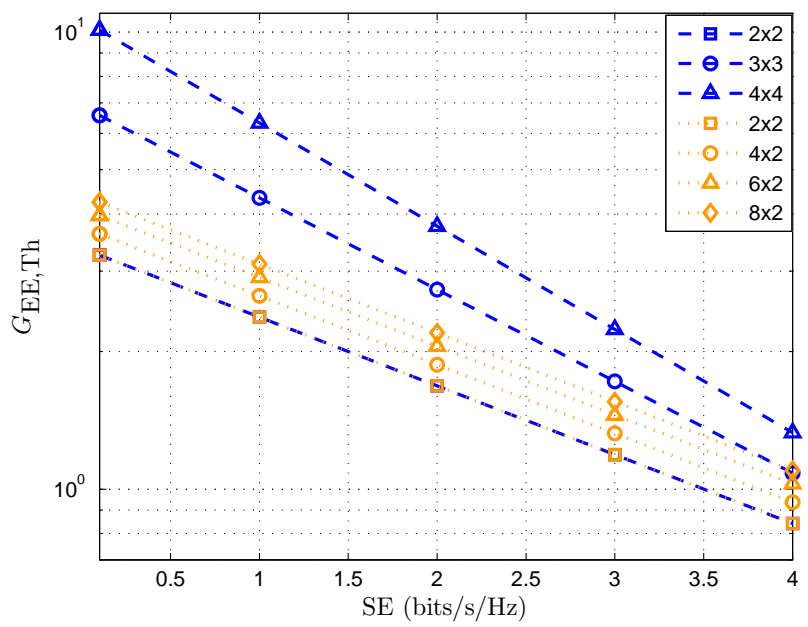

Fig. 6. Energy efficiency gain by using TAS/MRC instead of MIMO as a function of the spectral efficiency, for various numbers of antenna elements, by considering a theoretical power consumption model, where only the transmit power is taken into account.

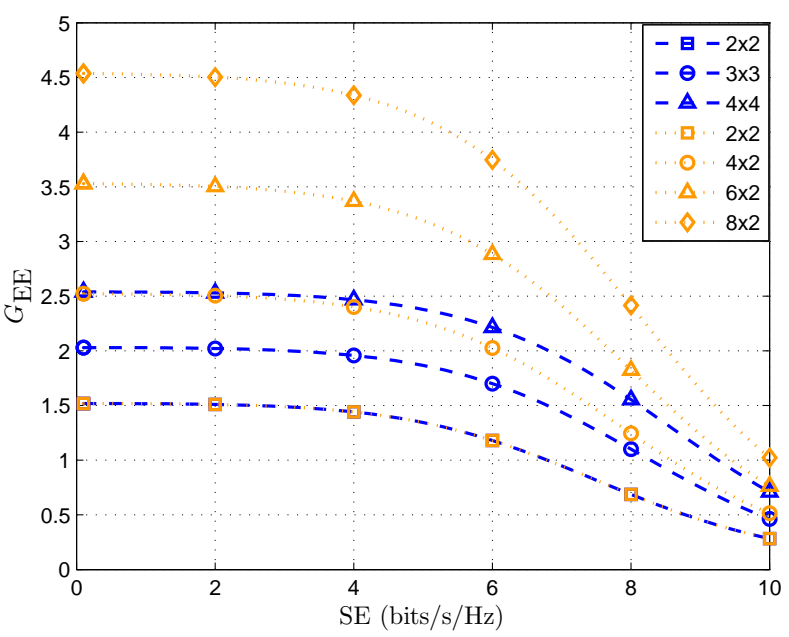

Fig. 7. Energy efficiency gain by using TAS/MRC instead of MIMO as a function of the spectral efficiency, for various numbers of antenna elements, by considering the double linear power consumption model, where the circuits power consumption is also taken into account.

circuitry is also taken into account. As expected, the gain decreases as the spectral efficiency increases. However, since the power consumed by the circuitry dominates the total power consumption for the low spectral efficiency region, in this situation the range in which the TAS/MRC scheme outperforms the MIMO scheme in terms of energy efficiency (the region where the gain is greater than one) is wider.

Fig. 8 shows the energy efficiency gain of TAS/MRC over MIMO considering the theoretical PCM. We can see that TAS/MRC scheme outperforms the MIMO scheme for low spectral efficiency values, regardless the number of antenna elements. As the spectral efficiency increases, as expected, the MIMO scheme is more energy efficient than TAS/MRC. In Fig. 9 we present results for the same setup as in Fig. 8, but considering the double linear PCM instead, where some interesting observations can be made. For instance, for spectral efficiency values in the order of $2.0 \mathrm{bits} / \mathrm{s} / \mathrm{Hz}$, the gain of TAS/MRC over MIMO keeps increasing with the number of 


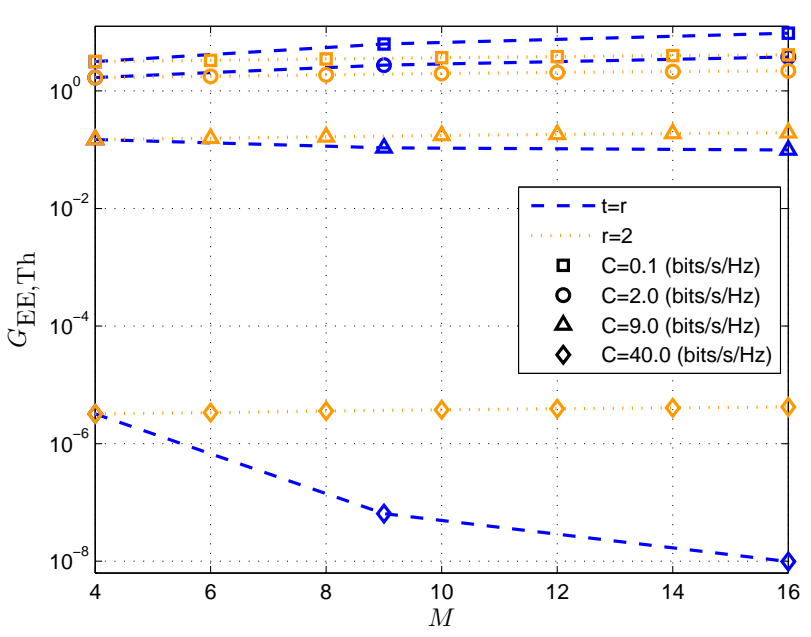

Fig. 8. Energy efficiency gain by using TAS/MRC instead of MIMO as a function of the number of antenna elements, for various spectral efficiency values, by considering a theoretical power consumption model, where only the transmit power is taken into account.

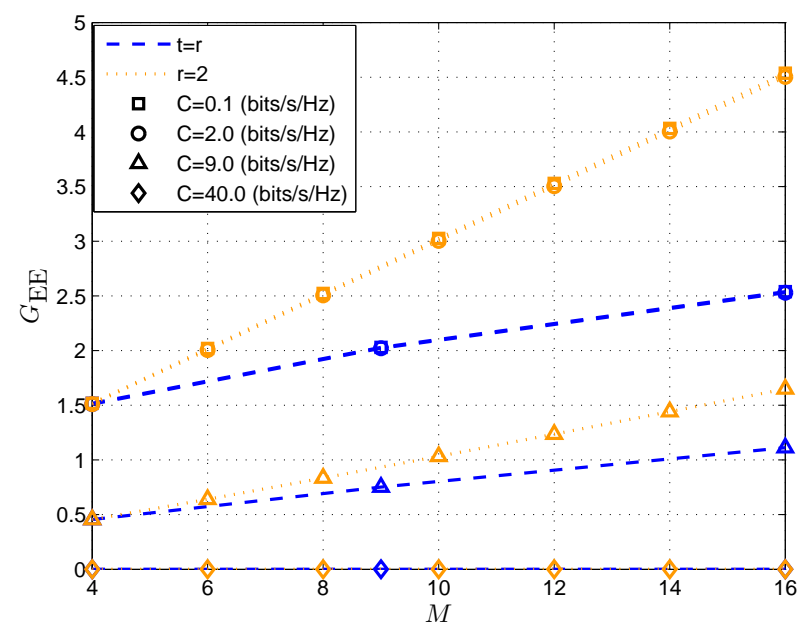

Fig. 9. Energy efficiency gain by using TAS/MRC instead of MIMO as a function of the number of antenna elements, for various spectral efficiency values, by considering the double linear power consumption model, where the circuits power consumption is also taken into account.

antenna elements, while in Fig. 8 this gain remains nearly constant in the same interval. Another interesting fact occurs at higher values of spectral efficiency, in the order of 9.0 $\mathrm{bits} / \mathrm{s} / \mathrm{Hz}$, where if we keep increasing the number of antennas, TAS/MRC becomes more energy efficient than MIMO.

Fig. 10 shows the upper bound for the $\mathrm{EE}$ as a function of the $P_{0} / P_{1}$ ratio, for a fixed $\mathrm{SE}$ of $7.0 \mathrm{bits} / \mathrm{s} / \mathrm{Hz}$. As expected, when the ratio $P_{0} / P_{1}$ grows, MIMO becomes less energy efficient than TAS/MRC, since $P_{0}$ is not multiplied by $t$ in (13). Also, by the same reason, when the number of antennas increases, the threshold from which TAS/MRC is more energy efficient than MIMO decreases. In this example, the $P_{0} / P_{1}$ thresholds where TAS/MRC becomes more energy efficient than MIMO are $122 \%, 36 \%$ and $17 \%$ for $M=4$, $M=9$ and $M=16$, respectively.

Finally, by fixing the number of receive antennas at $r=$ 2, we observe in Fig. 11 that the $P_{0} / P_{1}$ threshold where TAS/MRC becomes more energy efficient than MIMO is lower

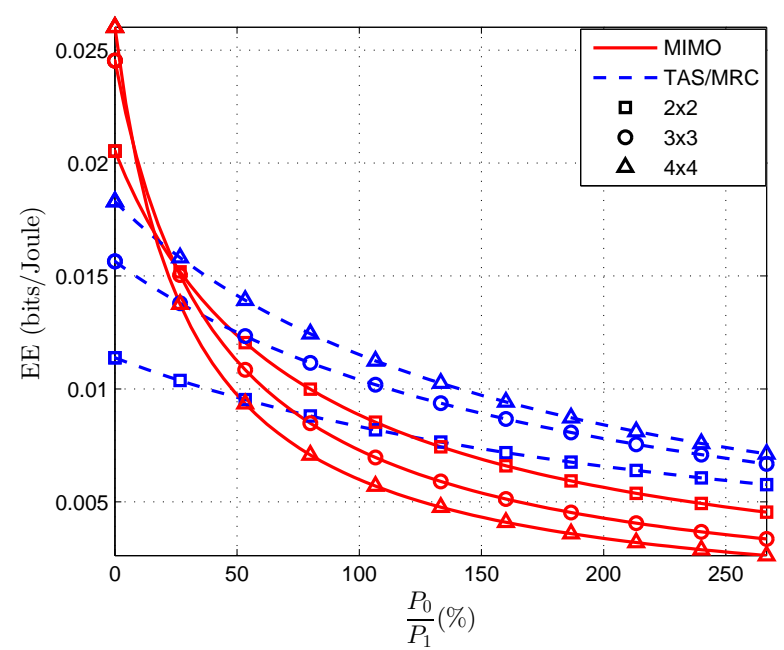

Fig. 10. Energy efficiency upper bound of TAS/MRC and MIMO schemes, as a function of the number of the ratio between the part of the overhead power that grows linearly with the number of antennas and the part that remains fixed, for a fixed spectral efficiency value $\mathrm{SE}=7.0 \mathrm{bits} / \mathrm{s} / \mathrm{Hz}$.

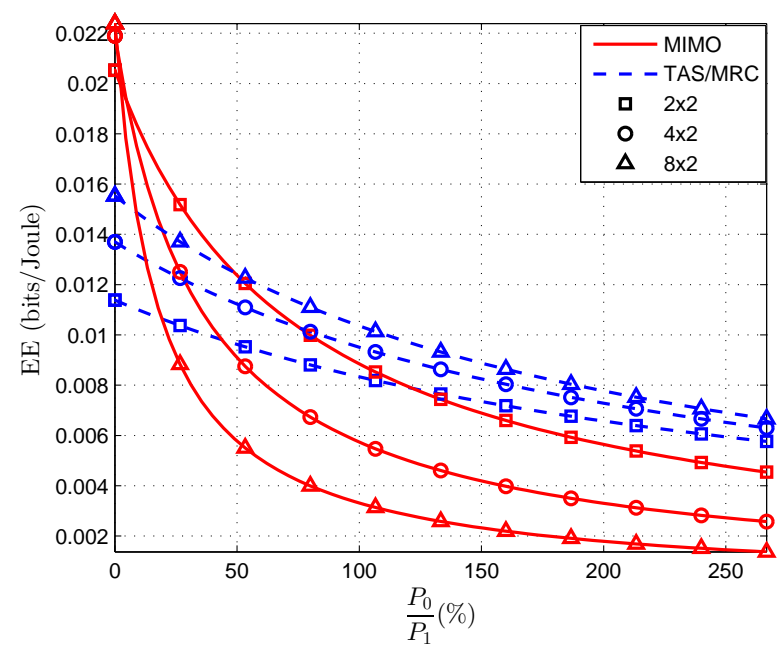

Fig. 11. Energy efficiency upper bound of TAS/MRC and MIMO schemes, as a function of the number of the ratio between the part of the overhead power that grows linearly with the number of antennas and the part that remains fixed, for a fixed spectral efficiency value $\mathrm{SE}=7.0 \mathrm{bits} / \mathrm{s} / \mathrm{Hz}$, with $r$ fixed.

than that of Fig. 10. This occurs since we increase $t$ as to maintain $M$ similar for both cases and, thus, once $P_{0}$ is multiplied by $t$ in the MIMO scenario, the energy consumption increases. In this example, the $P_{0} / P_{1}$ threshold is $28 \%$ for $M=8$, and $8.8 \%$ for $M=16$.

\section{Final Comments}

We evaluate the energy efficiency as a function of spectral efficiency and the number of antennas for TAS/MRC and MIMO schemes. Moreover, the optimal spectral efficiency value that maximizes the energy efficiency of the TAS/MRC scheme, for a given number of antennas, is obtained in closed-form and supported by numerical results. We show that TAS/MRC can be more energy efficient than MIMO in the low spectral efficiency region, and that such advantage increases with the number of antennas. Moreover, since placing many multiple antennas only at the transmitter side may 
be more feasible in practice, once many networks are composed of receivers with complexity/cost/physical dimensions limitations, we also show that the relative energy efficiency of TAS/MRC increases when compared to MIMO when the number of receive antennas is fixed at $r=2$. In addition, we also show the importance of considering a realistic power consumption model for the system analysis, since the gain obtained by choosing TAS/MRC instead of MIMO is lower if a theoretical PCM is considered. Our analysis also shows that if the ratio between the part of the overhead power that grows linearly with the number of antennas and the part that remains fixed increases, MIMO becomes less energy efficient than TAS/MRC, since the overhead power consumption of TAS/MRC does not scales with the number of antennas, as only one transmit antenna is selected at a time.

\section{REFERENCES}

[1] J. Mietzner, R. Schober, L. Lampe, W. Gerstacker, and P. Hoeher, "Multiple-antenna techniques for wireless communications - a comprehensive literature survey," IEEE Commun. Surveys Tuts., vol. 11, no. 2, pp. 87-105, quarter 2009.

[2] G. Auer, V. Giannini, C. Desset, I. Godor, P. Skillermark, M. Olsson, M. Imran, D. Sabella, M. Gonzalez, O. Blume, and A. Fehske, "How much energy is needed to run a wireless network?" IEEE Wireless Commun. Mag., vol. 18, no. 5, pp. 40-49, Oct. 2011.

[3] H. Kwon and T. Birdsall, "Channel capacity in bits per joule," IEEE J. Ocean. Eng., vol. 11, no. 1, pp. 97-99, Jan 1986.

[4] V. Rodoplu and T. Meng, "Bits-per-joule capacity of energy-limited wireless networks," IEEE Trans. Wireless Commun., vol. 6, no. 3, pp. 857-865, Mar. 2007.

[5] S. Verdu, "Spectral efficiency in the wideband regime," IEEE Trans. Inf. Theory, vol. 48, no. 6, pp. 1319-1343, Jun 2002.

[6] G. Brante, I. Stupia, R. D. Souza, and L. Vandendorpe, "Outage probability and energy efficiency of cooperative MIMO with antenna selection," IEEE Trans. Wireless Commun., vol. 12, no. 11, pp. 58965907, Nov. 2013.

[7] S. Cui, A. Goldsmith, and A. Bahai, "Energy-constrained modulation optimization," IEEE Trans. Wireless Commun., vol. 4, no. 5, pp. 2349 - 2360, Sept. 2005.

[8] T. Quek, D. Dardari, and M. Win, "Energy efficiency of dense wireless sensor networks: to cooperate or not to cooperate," IEEE J. Sel. Areas Commun., vol. 25, no. 2, pp. 459 - 470, Sept. 2007.

[9] G. Brante, M. T. Kakitani, and R. D. Souza, "Energy efficiency analysis of some cooperative and non-cooperative transmission schemes in wireless sensor networks," IEEE Trans. Commun., vol. 59, no. 10, pp. 2671-2677, Oct. 2011.

[10] O. Arnold, F. Richter, G. Fettweis, and O. Blume, "Power consumption modeling of different base station types in heterogeneous cellular networks," in Future Network and Mobile Summit, 2010, June 2010, pp. 1-8.

[11] A. Fehske, P. Marsch, and G. Fettweis, "Bit per joule efficiency of cooperating base stations in cellular networks," in IEEE GLOBECOM Workshops, Dec. 2010, pp. 1406-1411.

[12] F. Heliot, M. Imran, and R. Tafazolli, "On the energy efficiency gain of MIMO communication under various power consumption models," in Future Network Mobile Summit (FutureNetw), Jun. 2011, pp. 1 -9.

[13] G. Li, Z. Xu, C. Xiong, C. Yang, S. Zhang, Y. Chen, and S. Xu, "Energyefficient wireless communications: tutorial, survey, and open issues," IEEE Wireless Commun. Mag., vol. 18, no. 6, pp. 28 -35, Dec. 2011.

[14] F. Heliot, M. Imran, and R. Tafazolli, "On the energy efficiency-spectral efficiency trade-off over the MIMO Rayleigh fading channel," IEEE Trans. Commun., vol. 60, no. 5, pp. 1345-1356, May 2012.

[15] J. Jiang, M. Dianati, M. Imran, R. Tafazolli, and Y. Chen, "On the relation between energy efficiency and spectral efficiency of multipleantenna systems," IEEE Trans. Veh. Technol., vol. 62, no. 7, pp. 3463 3469, Sept 2013.

[16] O. Onireti, F. Heliot, and M. Imran, "On the energy efficiency-spectral efficiency trade-off of distributed MIMO systems," IEEE Trans. Commun., vol. 61, no. 9, pp. 3741-3753, Sep. 2013.
[17] I. Ku, C.-X. Wang, and J. Thompson, "Spectral-energy efficiency tradeoff in relay-aided cellular networks," IEEE Trans. Wireless Commun., vol. 12, no. 10, pp. 4970-4982, Oct. 2013.

[18] S. Huang, H. Chen, J. Cai, and F. Zhao, "Energy efficiency and spectralefficiency tradeoff in amplify-and-forward relay networks," IEEE Trans. Veh. Commun., vol. 62, no. 9, pp. 4366-4378, Nov. 2013.

[19] J. Wu, G. Wang, and Y. Zheng, "Energy efficiency and spectral efficiency tradeoff in type-i ARQ systems," IEEE J. Sel. Areas Commun., vol. 32, no. 2, pp. 356-366, Feb. 2014.

[20] M. K. Simon and M. S. Alouini, Digital Communication over Fading Channels. Wiley Interscience, 2004.

[21] M. T. Kakitani, G. Brante, R. D. Souza, and M. A. Imran, "Energy efficiency of transmit diversity systems under a realistic power consumption model," IEEE Commun. Lett., vol. 17, no. 1, pp. 119-122, Jan. 2013.

[22] H. Li, L. Song, and M. Debbah, "Energy efficiency of large-scale multiple antenna systems with transmit antenna selection," IEEE Trans. Commun., vol. 62, no. 2, pp. 638-647, Feb. 2014.

[23] A. Goldsmith, Wireless Communications. Cambridge University Press, 2005.

[24] C. Shannon, "A mathematical theory of communications," Bell Syst. Tech. J., vol. 27, pp. 379-423 e 623-656, 1948.

[25] I. Gradshteyn and I. Ryzhik, Table of Integrals, Series, and Products, 7th ed., A. Jeffrey and D. Zwillinger, Eds. Academic Press, 2007.

[26] X. Zhang, Z. Lv, and W. Wang, "Performance analysis of multiuser diversity in mimo systems with antenna selection," IEEE Trans. Wireless Commun., vol. 7, no. 1, pp. 15-21, Jan 2008.

[27] R. M. Corless, G. H. Gonnet, D. E. G. Hare, D. J. Jeffrey, and D. E. Knuth, "On the Lambert W function," Advances in Computational Mathematics, vol. 5, no. 1, pp. 329-359, Dec. 1996. 